\title{
Quantized conductance through a dissipative atomic point contact
}

\section{Journal Article}

\section{Author(s):}

Corman, Laura; Fabritius, Philipp; Häusler, Samuel; Mohan, Jeffrey (D); Dogra, Lena H.; Husmann, Dominik; Lebrat, Martin; Esslinger, Tilman

\section{Publication date:}

2019-11

\section{Permanent link:}

https://doi.org/10.3929/ethz-b-000379510

\section{Rights / license:}

In Copyright - Non-Commercial Use Permitted

\section{Originally published in:}

Physical Review A 100(5), https://doi.org/10.1103/physreva.100.053605

\section{Funding acknowledgement:}

182650 - Interplay between Topology, Interactions and Dissipation in Driven Quantum Many-Body Systems (SNF)

742579 - Mass, heat and spin transport in interlinked quantum gases (EC)

746150 - Topological states with Spin-Dependent potentials for ultracold lithium (EC) 


\title{
Quantized conductance through a dissipative atomic point contact
}

\author{
Laura Corman $\odot,{ }^{1,}$ ' Philipp Fabritius $\odot,{ }^{1}$ Samuel Häusler, ${ }^{1}$ Jeffrey Mohan $\odot,{ }^{1}$ Lena H. Dogra $\odot,{ }^{1,2}$ \\ Dominik Husmann ${ }^{\circ},{ }^{1}$ Martin Lebrat, ${ }^{1}$ and Tilman Esslinger ${ }^{1}$ \\ ${ }^{1}$ Department of Physics, ETH Zürich, 8093 Zürich, Switzerland \\ ${ }^{2}$ Cavendish Laboratory, University of Cambridge, J. J. Thomson Avenue, Cambridge CB3 OHE, England, United Kingdom
}

(Received 7 July 2019; published 8 November 2019)

\begin{abstract}
Signatures of quantum transport are expected to quickly vanish as dissipation is introduced in a system. This dissipation can take several forms, including that of particle loss, which has the consequence that the total probability current is not conserved. Here, we study the effect of such losses at a quantum point contact (QPC) for ultracold atoms. Experimentally, dissipation is provided by a near-resonant optical tweezer the power and detuning of which control the loss rates for the different internal atomic states as well as their effective Zeeman shifts. We theoretically model this situation by including losses in the Landauer-Büttiker formalism over a wide range of dissipative rates. We find good agreement between our measurements and our model, both featuring robust conductance plateaus. Finally, we are able to map out the atomic density by varying the position of the near-resonant tweezer inside the QPC, realizing a dissipative scanning gate microscope for cold atoms.
\end{abstract}

DOI: 10.1103/PhysRevA.100.053605

\section{INTRODUCTION}

Coupling a system to its environment is a central concept in physics, giving rise to different types of ensembles in thermodynamics [1]. It leads to fundamental questions about the fate of quantum mechanics at a macroscopic scale [2,3]. The coupling to the environment can also compete with coherence and interaction effects, leading to new phenomena [4,5]. A full model of a system and its environment is out of reach in most cases due to the exponential growth of the total Hilbert space with the number of degrees of freedom. Tracing out those of the environment can be done under certain assumptions leading to a Lindblad master equation [6]. In some cases, further simplification is possible by only adding dissipative terms to the isolated system's equation of motion $[7,8]$. The resulting dynamics, which becomes non-Hermitian, has recently found renewed interest due to the exotic behaviors of exceptional points linked to the collapse of the eigenvectors at a critical dissipation strength $[9,10]$.

Dissipation, understood as the nonconservation of the system's volume in phase space over time, is characteristic for transport phenomena where currents bring a system towards equilibrium while producing entropy [1]. There, extrinsic dissipation can be included by physical or fictitious coupling to a reservoir other than the leads driving the transport processes. Such an additional coupling has mostly been studied to model incoherent scattering of electrons [11-13]. It is usually done by adding a fictitious reservoir with which the lossy region can exchange particles without any net current [11]. Mimicking particle losses as a perturbation to transport by an additional absorbing reservoir has to our knowledge so far not been explored in this context.

\footnotetext{
*Corresponding author: 1corman@ phys.ethz.ch
}

Cold clouds of atoms are nearly closed systems by default, but can be opened by designing atom losses in a controlled way. Experimental loss channels include (i) molecule formation via photoassociation [14] or via decay to a molecular channel [15-17], (ii) ionization using a focused electron beam $[18,19]$ or a femtosecond laser [20], or (iii) scattering of near-resonant photons that impart a large kinetic energy to the atoms [21-23]. Many of these techniques realize localized losses, an assumption underlying the usual theoretical models for dissipation in transport structures [13], realized using an electron beam in a Bose-Einstein condensate [24,25].

In this paper, we study dissipation as a perturbation to transport through a quantum point contact (QPC) for a twocomponent fermionic gas of ultracold ${ }^{6} \mathrm{Li}$ atoms, where the different hyperfine states of the atoms are interpreted as a pseudospin. The geometry of the optical potentials trapping the atoms realizes a two-terminal transport setup as illustrated in Fig. 1(a) [26]. There, a cloud of degenerate fermionic lithium is divided into two reservoirs connected by a quasione-dimensional (1D) constriction formed by two intersecting beams with a nodal line. Controlled by the chemical potential inside the 1D channel, only one to two transverse modes are available to particles moving from one reservoir to the other. Since the potential landscape in the channel is smooth, transport is ballistic: the transmission probability of the atoms is close to unity. This leads to the measurement of conductance plateaus [27] as the chemical potential is increased in the wire, in agreement with the Landauer-Büttiker model [illustrated in Fig. 1(b)] that identifies conductance with transmission for mesoscopic conductors [28-30].

The losses are provided by a near-resonant beam focused onto the constriction. The spatial profile of the beam is controlled holographically using a digital micromirror device, allowing us to correct for aberrations [31]. The scattering rate and dipole potential experienced by the atoms are related to the imaginary and real part of the atomic polarizability, 
(a)

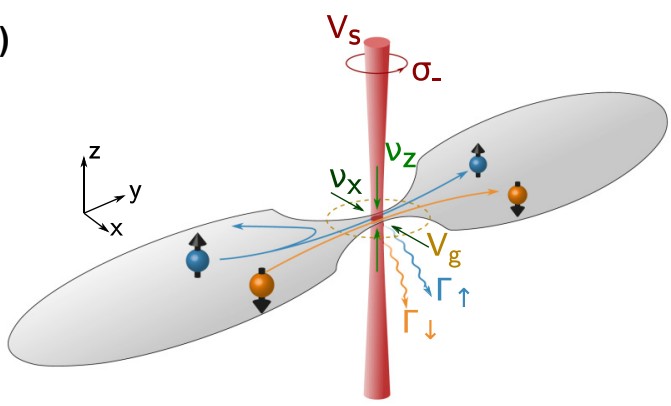

(b)

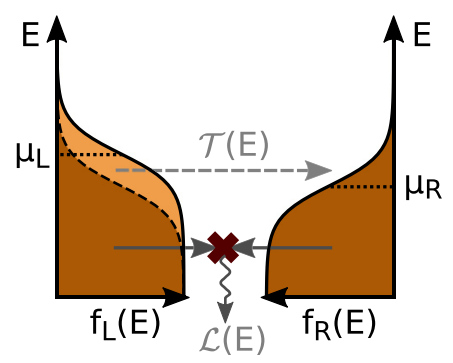

FIG. 1. Experimental realization of a Landauer-Büttiker setup with losses. (a) ${ }^{6} \mathrm{Li}$ atoms in the lowest $(|\downarrow\rangle$, orange) and thirdlowest $(|\uparrow\rangle$, blue) hyperfine states are allowed to flow between two reservoirs connected by an optically defined QPC (gray). A nearresonant optical tweezer (red) with waist $w_{s}=2.0(1) \mu \mathrm{m}$ introduces different loss rates $\Gamma_{\uparrow}$ and $\Gamma_{\downarrow}$ as well as a spin-dependent potential $V_{s}$ inside the QPC. A far-detuned gate beam (dashed circle) locally increases the chemical potential $\mu_{\text {res }}$ imposed by the reservoirs by $V_{g}$. (b) In a Landauer-Büttiker picture and in the absence of a temperature bias, net transport can be attributed to the excess of particles in the left reservoir not compensated by the right reservoir (orange) due to a chemical potential difference $\mu_{L}-\mu_{R}>0$. Losses on the other hand involve the energy integration of the reservoir Fermi-Dirac distributions $f_{L}(E)$ and $f_{R}(E)$, including states below the Fermi level that do not contribute to a net current (brown). Conductance and loss are furthermore weighted by the energy-dependent transmission $\mathcal{T}(E)$ and loss $\mathcal{L}(E)$ coefficients of the mesoscopic channel.

respectively, which must be computed in the regimes of high magnetic fields relevant for tuning the scattering properties of ${ }^{6} \mathrm{Li}$. For light frequencies close to the atomic resonances of different pseudospin states, both scattering rate and dipole potential are strongly spin dependent due to the splitting of their transition frequencies. The dipole potential can be interpreted as an effective Zeeman shift in the case where its magnitudes are equal and opposite for both spins. This case is investigated in our companion paper [32] wherein the amplitude of this effective Zeeman shift is on the order of the Fermi energy of the atoms, leading to a significant shift between the onset of the conductance plateaus of the two spins. Here, we focus on the effect of the atom losses engineered for the atoms. We demonstrate that in spite of the dissipation the conductance plateaus persist owing to the constant flow of low-temperature particles through the channel. We show that the transport properties of a lossy QPC can be described by including the transmission as well as the energy-dependent losses characterizing the channel into a Landauer-Büttiker model as illustrated in Fig. 1(b). Finally, we are able to reconstruct the atomic density in a wide region around the QPC by monitoring the atom losses as the position of the near-resonant tweezer is varied.

This paper is structured as follows. In Sec. II, we review the polarizability of alkali-metal atoms in high magnetic fields. In Sec. III, we adapt the Landauer formalism to the presence of particle losses. In Sec. IV, we describe the experimental setup and the loss mechanism induced by the near-resonant light, justifying the validity of the analysis of Sec. III. Finally, we compare the extended Landauer formalism to our measurements in Sec. V.

\section{II. ${ }^{6}$ Li POLARIZABILITY IN HIGH MAGNETIC FIELDS}

In the following, we briefly review how to compute the atomic polarizability in presence of a high magnetic field, which is particularly relevant when the interaction strength can be controlled using a Feshbach resonance such as ${ }^{6} \mathrm{Li}$. Choosing the frequency and intensity of a near-resonant beam allows to tailor different optical potentials and losses for each internal state of ultracold lithium atoms. We will focus on presenting regimes where the optical potentials are of equal magnitude but opposite sign for the two relevant states in the experiment, emulating an effective Zeeman shift.

\section{A. Polarizability of alkali-metal atoms in the Paschen-Back regime}

We consider alkali-metal atoms in a uniform magnetic field $\vec{B}$. If the energy of the electron's magnetic dipole moment exceeds the hyperfine interaction for ${ }^{6} \mathrm{Li}$, the atoms are in the Paschen-Back regime.

The Hamiltonian for a single atom can be expressed as a function of the electronic and nuclear spin operators $\hat{\vec{J}}=\hat{\vec{L}}+$ $\hat{\vec{S}}$ and $\hat{\vec{I}}$ :

$$
\hat{H}_{\mathrm{at}}=\frac{\mu_{B}}{\hbar}\left(g_{J} \hat{\vec{J}} \cdot \vec{B}+g_{I} \hat{\vec{I}} \cdot \vec{B}\right)+\frac{a}{\hbar^{2}} \hat{\vec{J}} \cdot \hat{\vec{I}},
$$

where $\mu_{B}$ is the Bohr magneton, $g_{J}$ and $g_{I}$ are the electronic and nuclear gyromagnetic factors, and $a$ is the hyperfine coupling energy. Both $g_{J}$ and $a$ depend on the electron's orbital momentum $L$ and total spin $J$ which characterize each level manifold $2 S, 2 P_{1 / 2}$, and $2 P_{3 / 2}$. Their values for ${ }^{6} \mathrm{Li}$ are reported in [33].

The dipole matrix element between two states labeled by electron and nuclear spin $\left(J, m_{J}, m_{I}\right)$ is given by the WignerEckardt theorem:

$$
d_{k k^{\prime}}^{q}=\frac{1}{\sqrt{2 J^{\prime}+1}} \delta_{m_{I}, m_{I}^{\prime}}\left\langle J m_{J}, 1 q \mid J^{\prime} m_{J}^{\prime}\right\rangle\left\langle J^{\prime}|| \vec{d}|| J\right\rangle,
$$

where $\left\langle J m_{J}, 1 q \mid J^{\prime} m_{J}^{\prime}\right\rangle$ is the Clebsch-Gordan coefficient relating initial and final electron spins $m_{J}$ and $m_{J}^{\prime}$ through the added angular momentum $q=-1,0$, and +1 of a photon with polarization $\sigma^{-}, \pi$, and $\sigma^{+}$, respectively, and $\left\langle J^{\prime}|| \vec{d}|| J\right\rangle$ is the reduced matrix element of the corresponding $2 S \rightarrow 2 P$ transition. The dipole matrix elements $d_{e g}^{q}$ between each pair of eigenstates $|g\rangle$ and $|e\rangle$ of (1) are then given via a change of basis.

These dipole matrix elements appear in the complex polarizability tensor of each state $|g\rangle$ of the ground manifold $2 S$ at 
light frequency $v$ :

$$
\alpha_{g}^{q q^{\prime}}=-\sum_{e} \frac{\left(d_{e g}^{q}\right)^{*} d_{e g}^{q^{\prime}}}{h\left(v-v_{e g}\right)+i \hbar \Gamma_{e g} / 2},
$$

where the sum runs over all excited states $|e\rangle$ in $2 P_{1 / 2}$ and $2 P_{3 / 2}$. The transition frequencies $v_{e g}$ between ground and excited states are derived from the eigenvalues of (1), and $\Gamma_{e g}$ denotes the spontaneous emission rates from excited to ground states:

$$
\Gamma_{e g}=\frac{\left(h v_{e g}\right)^{3}}{3 \pi \epsilon_{0} c^{3} \hbar^{4}} \sum_{q}\left|d_{e g}^{q}\right|^{2},
$$

where $c$ is the speed of light and $\epsilon_{0}$ is the vacuum permittivity.

For light intensities $I$ small compared to the saturation intensity, the light shift $V_{|g\rangle}$ and scattering rate $\Gamma_{|g\rangle}$ experienced in the ground state $|g\rangle$ are related to the dispersive and dissipative parts of the polarizability:

$$
\begin{gathered}
V_{|g\rangle}=-\frac{I}{2 \epsilon_{0} c} \sum_{q, q^{\prime}} e_{q} \operatorname{Re}\left(\alpha_{g}^{q q^{\prime}}\right) e_{q^{\prime}}^{*}, \\
\Gamma_{|g\rangle}=\frac{I}{\hbar \epsilon_{0} c} \sum_{q, q^{\prime}} e_{q} \operatorname{Im}\left(\alpha_{g}^{q q^{\prime}}\right) e_{q^{\prime}}^{*}
\end{gathered}
$$

The sum indicates a double tensor contraction by the light polarization $\vec{e}=\vec{E} /|\vec{E}|=\sum_{q} e_{q} \vec{u}_{q}$, the coordinates of which are expressed here in the basis $\vec{u}_{-1}=\left(\vec{u}_{x}-i \vec{u}_{y}\right) / \sqrt{2}$ for $\sigma^{-}$ polarization, $\vec{u}_{0}=\vec{u}_{z}$ for $\pi$ polarization, and $\vec{u}_{+1}=-\left(\vec{u}_{x}+\right.$ $\left.i \vec{u}_{y}\right) / \sqrt{2}$ for $\sigma^{+}$polarization.

\section{B. Spin filter regimes for ultracold ${ }^{6} \mathrm{Li}$ atoms}

The eigenstates of the Hamiltonian (1) can be expressed as linear combinations of the $\left|J, m_{J}, m_{I}\right\rangle$ states. From an experimental point of view, we are especially interested in the lowest and third-lowest hyperfine states as possible ground states, labeled as $|\downarrow\rangle$ and $|\uparrow\rangle$. by

At high magnetic field $B$, these states can be approximated

$$
\begin{gathered}
|\downarrow\rangle=\left|m_{J}=-\frac{1}{2}, m_{I}=1\right\rangle-\frac{\epsilon}{\sqrt{2}}\left|m_{J}=\frac{1}{2}, m_{I}=0\right\rangle, \\
|\uparrow\rangle=\left|m_{J}=-\frac{1}{2}, m_{I}=-1\right\rangle
\end{gathered}
$$

to first order in $\epsilon=a h /\left(g_{J} \mu_{B} B\right)$, with $a=152.1 \mathrm{MHz}$ the hyperfine coupling constant of the $2^{2} S_{1 / 2}$ manifold.

Therefore the different internal states are mostly described by their electronic spin $m_{J}=-1 / 2$ and nuclear spin, $m_{I}=$ +1 and -1 , for the lowest and third-lowest hyperfine states of ${ }^{6} \mathrm{Li}$, respectively.

For a given polarization, the transitions between these hyperfine states and the excited manifolds $2 P_{1 / 2}$ and $2 P_{3 / 2}$ occur at different frequencies, typically offset by a magneticfield-dependent shift comparable to the hyperfine coupling. Tuning the light frequency in the vicinity of these transitions leads to strongly state-dependent polarizabilities which can be exploited experimentally.

As an example, and along the lines of our companion paper [32], the differential light shift created by a near-resonant beam can be reinterpreted as an effective Zeeman shift. We therefore aim at creating the maximum potential difference $V_{\uparrow}-V_{\downarrow}$ for a limited scattering rate at a frequency where the mean potential for the two spin states vanishes $\left(V_{\downarrow}+V_{\uparrow}\right) / 2=0$.

A natural and experimentally practical solution adopted in [32] is to choose a frequency $v$ right in between the strongest transitions of the states $|\downarrow\rangle$ and $|\uparrow\rangle$ to the $D_{2}$ line, which fulfill the condition $m_{J^{\prime}}=m_{J}+q$ and the linewidths of which are almost equal at high magnetic field. For $\sigma^{-}$light, the resonance frequencies $\nu_{\downarrow}$ and $\nu_{\uparrow}$ are $162.6 \mathrm{MHz}$ apart at the typical magnetic field of $B=568 \mathrm{G}$ where the scattering length between the two spin states vanishes. The potentials and scattering rates for each atomic internal state are displayed in Figs. 2(a) and 2(b) as a function of the mean detuning $\bar{\delta}=v-\left(v_{\downarrow}+v_{\uparrow}\right) / 2$.

Alternative solutions are, however, more favorable in terms of light scattering. Interestingly, the small admixture of $m_{J}=$ $1 / 2$ states in $|\downarrow\rangle$ as seen in Eq. (7) leads to narrow transitions to the states $m_{J^{\prime}}=1 / 2+q$ with an effective width $\sim \epsilon^{2} \Gamma / 2$. Figures 2(c) and 2(d) shows the dipole potential and scattering rate close to such a transition for $\sigma^{+}$polarization within the $D_{2}$ line, adapting the light intensity such that the differential light shift remains equal to the one in Fig. 2(a). The associated scattering rate is reduced by more than one order of magnitude compared to Fig. 2(b).

Last, the mean potential also vanishes at a frequency between the $D_{1}$ and the $D_{2}$ lines for $\sigma^{+}$and $\pi$ polarization. As illustrated in Figs. 2(e) and 2(f) for $\sigma^{+}$polarization, the signs of the dipole potentials $V_{\uparrow}$ and $V_{\downarrow}$ are inverted compared to the two previous cases. The scattering rate at fixed differential light shift is only reduced by a factor of 2 , but it is equal for the two spin states and remains weakly frequency dependent.

\section{ADAPTING THE LANDAUER FORMALISM WITH LOSSES}

Transport between two reservoirs through a QPC is usually well described by Landauer's formalism which identifies conductance with transmission [28-30]. Our aim here is to extend this formalism to localized particle losses by modeling them by an imaginary potential.

\section{A. Effective Hamiltonian with losses}

For a system evolving according to a Hamiltonian $\hat{H}_{0}$ which is weakly coupled to a fast evolving environment (Born-Markov approximation), the evolution of the density matrix $\rho$ is determined by the Lindblad master equation [6]:

$$
\partial_{t} \hat{\rho}=-\frac{i}{\hbar}[\hat{H}, \hat{\rho}]-\sum_{j}\left(\frac{1}{2} \hat{L}_{j}^{\dagger} \hat{L}_{j} \hat{\rho}+\frac{1}{2} \hat{\rho} \hat{L}_{j}^{\dagger} \hat{L}_{j}-\hat{L}_{j} \hat{\rho} \hat{L}_{j}^{\dagger}\right)
$$

where $\hat{L}_{j}$ represent the noise operators describing the loss processes, which are proportional to the square root of the scattering rate (6). The first two terms of the sum can be included in the commutator by defining an effective, complexvalued Hamiltonian

$$
\hat{H}_{\text {eff }}=\hat{H}_{0}-i \frac{\hbar}{2} \sum_{j} \hat{L}_{j}^{\dagger} \hat{L}_{j}
$$




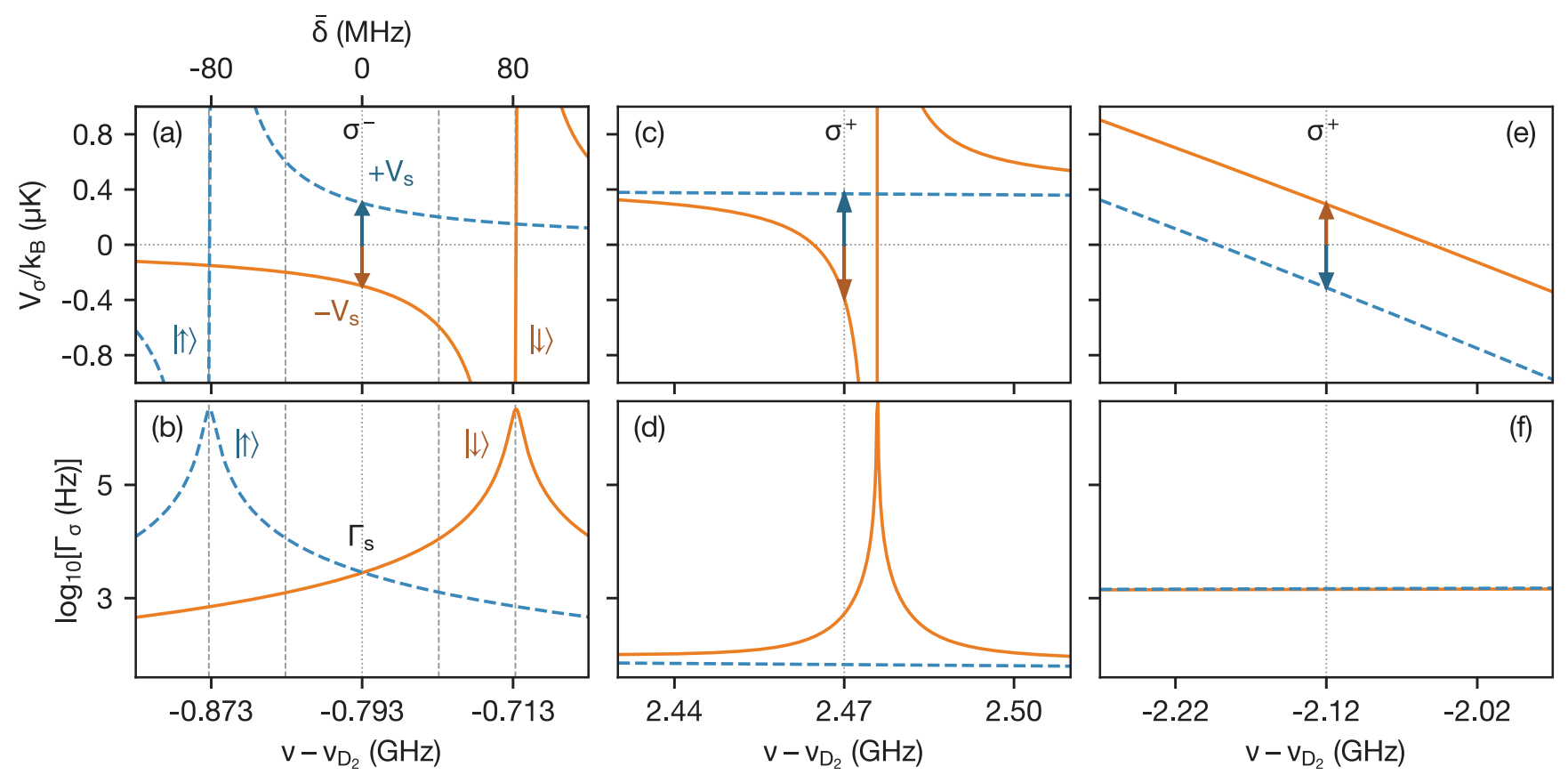

FIG. 2. Potentials and scattering rates at a magnetic field of $B=568 \mathrm{G}$ for the $|\downarrow\rangle$ and $|\uparrow\rangle$ states for different frequencies $v$ of the light where the mean potential $\left(V_{\downarrow}+V_{\uparrow}\right) / 2$ vanishes. The lower frequency axes are offset by the $D_{2}$ transition frequency at zero magnetic field $v_{D_{2}}$ [33]. The intensities in each pair of graphs are chosen to give a constant amplitude of the spin potential $\left|V_{\downarrow}-V_{\uparrow}\right|=k_{B} \times 0.6 \mu \mathrm{K}$. Panels (a) and (b) correspond to $\sigma^{-}$polarization and an intensity of $3 \mathrm{~W} / \mathrm{m}^{2}$. The top frequency axis has been offset by the mean of the resonance frequencies of state $|\downarrow\rangle$ and $|\uparrow\rangle$ for the main $D_{2} \sigma^{-}$transition: $\bar{\delta}=v-\left(v_{\downarrow}+v_{\uparrow}\right) / 2$. Panels (c) and (d) correspond to $\sigma^{+}$polarization and an intensity of $136 \mathrm{~W} / \mathrm{m}^{2}$ close to a narrow $D_{2}$ resonance appearing in the Paschen-Back regime. Panels (e) and (f) correspond to $\sigma^{+}$polarization and an intensity of $1.2 \times 10^{4} \mathrm{~W} / \mathrm{m}^{2}$ between the $D_{1}$ and $D_{2}$ lines.

which, for losses spatially varying along $y$, becomes

$$
\hat{H}_{\mathrm{eff}}=\hat{H}_{0}-i \frac{\hbar \Gamma(y)}{2} \hat{\Psi}^{\dagger}(y) \hat{\Psi}(y)
$$

where $\hat{\Psi}(y)$ is the particle annihilation operator at position $y$.

The last term in the sum of (9) represents fluctuations and is necessary to preserve fermionic commutation relations. Using an imaginary potential is a minimal way to capture non-Hermitian dynamics [9] that neglects this last term. This ignores the stochastic nature of the loss processes and applies well to particles described by a macroscopic wave function such as photons or condensed bosons [19,34]. Although unpaired fermions are not described by a macroscopic wave function, the last term of (9) can still be neglected in our case since it will not contribute to macroscopic response functions measured over times very large compared to the fluctuation timescales. Therefore, the non-Hermitian Hamiltonian approach (11) should be sufficient to model the conductance measurement in a transport experiment, performed for durations much longer than the typical scattering time $\Gamma^{-1}$.

\section{B. Transmission and loss coefficients}

In the ballistic regime, the conductance between two reservoirs at chemical potentials $\mu_{L}$ and $\mu_{R}$ only depends on the overall transmission at a given energy $E$ :

$$
\mathcal{T}(E)=\sum_{\operatorname{modes} \mathbf{n}} \mathcal{T}_{\mathbf{n}}(E)
$$

where $\mathcal{T}_{\mathbf{n}}(E)$ is the transmission of transverse mode $\mathbf{n}$, i.e., the probability that a particle injected at one end with energy $E$ will be transmitted to the other end.

The different transverse modes of the QPC are characterized by the quantum numbers $\mathbf{n}=\left(n_{x}, n_{z}\right)$ describing the transverse wave function along the harmonically confined directions. We will restrict the analysis to the first transverse mode $\mathbf{n}=(0,0)$, which is valid at low chemical potentials in the reservoirs.

The transmission $\mathcal{T}(E)$ is computed from the longitudinal, complex-valued, spin-dependent potential $V_{\sigma}(y)$ that the particles experience as they travel along the QPC region. To this end, forward and backward scattering amplitudes associated with this complex potential are computed by solving the time-independent 1D Schrödinger equation with Numerov's method [35]. Their square moduli are equal to the energydependent transmission $\mathcal{T}(E)$ and reflection $\mathcal{R}(E)$, respectively, and do not sum up to one since the total probability amplitude is not conserved by the nonunitary evolution. The loss probability $\mathcal{L}(E)$ is defined as the missing probability:

$$
\mathcal{T}(E)+\mathcal{R}(E)+\mathcal{L}(E)=1
$$

\section{Landauer-Büttiker formula}

In the Landauer-Büttiker picture presented in Fig. 1(b), transport arises from the sum of a right-moving and a leftmoving current of particles with the Fermi-Dirac distributions $f_{L}(E)$ and $f_{R}(E)$ of the left and right reservoirs, respectively, 
defined as

$$
f(E, \mu, T)=\frac{1}{1+e^{\frac{E-\mu}{k_{B} T}}}
$$

where $\mu=\mu_{L / R}$ and $T=T_{L / R}$ are the chemical potentials and temperatures of each reservoir.

The number of right and left movers that are transmitted through the mesoscopic channel with an energy-dependent probability $\mathcal{T}(E)$ per unit time is

$$
\dot{N}_{\rightarrow / \leftarrow}^{\text {trans }}=\frac{1}{h} \int_{-\infty}^{+\infty} f_{L / R}(E) \mathcal{T}(E) d E .
$$

This formula is independent of the fermions' velocity at a given energy $E$ thanks to the expression of the onedimensional density of states (see Sec. 2.1 in [36]). The total number of atoms crossing the channel per unit time is therefore

$$
\begin{aligned}
\dot{N}^{\text {trans }}= & \dot{N}_{\rightarrow}^{\text {trans }}+\dot{N}_{\leftarrow}^{\text {trans }} \\
= & \dot{N}_{\rightarrow}^{\text {trans }}-\dot{N}_{\leftarrow}^{\text {trans }} \\
& +2 \dot{N}_{\leftarrow}^{\text {trans }} .
\end{aligned}
$$

The net current is the difference between the right and left movers as in Eq. (17), corresponding to the orange shaded area in Fig. 1(b):

$$
\dot{N}_{\mathrm{c}}^{\text {trans }}=I_{N}=\frac{1}{h} \int_{-\infty}^{+\infty}\left[f_{L}(E)-f_{R}(E)\right] \mathcal{T}(E) d E
$$

where the subscript "c" stands for "contributing" to transport. The rest of the atoms are transmitted through the channel without contributing to the net current with a rate

$$
\dot{N}_{\text {nc }}^{\text {trans }}=2 \dot{N}_{\leftarrow}^{\text {trans }}
$$

(with a subscript "nc" for "noncontributing"). This corresponds to the brown shaded area in Fig. 1(b).

Particle transport is also associated with energy transport, leading to weak thermoelectric effects that are neglected in the rest of the paper [37]. Assuming that the reservoirs have the same temperature $T$, conductance is obtained using Ohm's law:

$$
I_{N}=G \Delta \mu \text {. }
$$

To compute conductance in Sec. V, we furthermore assume small biases $\Delta \mu$ relative to temperature $k_{B} T$ that simplify the expression to

$$
G=\frac{1}{h} \int_{-\infty}^{+\infty} \mathcal{T}(E)\left(-\frac{\partial f(E, \bar{\mu}, T)}{\partial E}\right) d E
$$

with $\bar{\mu}=\left(\mu_{L}+\mu_{R}\right) / 2$.

\section{Time evolution of the reservoir properties}

This Landauer-Büttiker picture can be refined to estimate the particle and energy losses occurring during transport.

In contrast to the net current (19), the absolute particle number loss is equal to the sum of the currents lost from the mesoscopic channel by photon scattering with probability $\mathcal{L}(E)$ :

$$
-\frac{d N}{d t}=\frac{1}{h} \int_{-\infty}^{+\infty}\left[f_{L}(E)+f_{R}(E)\right] \mathcal{L}(E) d E
$$

$$
\begin{aligned}
=\frac{1}{h} & \int_{-\infty}^{+\infty}\left[f_{L}(E)-f_{R}(E)\right] \mathcal{L}(E) d E \\
& +\frac{2}{h} \int_{-\infty}^{+\infty} f_{R}(E) \mathcal{L}(E) d E .
\end{aligned}
$$

Similar to Eqs. (15)-(20), the total rate of particle losses (23) can be split into a contributing term $\dot{N}_{\mathrm{c}}^{\text {loss }}$ corresponding to the atoms at the Fermi level in Eq. (24) [orange area in Fig. 1(b)] and a noncontributing term $\dot{N}_{\text {nc }}^{\text {loss }}$ corresponding to the atoms below the lowest Fermi level in Eq. (25) [brown area in Fig. 1(b)].

The time evolution of each reservoir, characterized by its particle number $N_{L, R}(t)$ and internal energy $U_{L, R}(t)$, can be evaluated iteratively by (i) converting the extensive quantities $\left(N_{L, R}, U_{L, R}\right)$ into chemical potential and temperature $\left(\mu_{L, R}, T_{L, R}\right)$ using the equation of state of the noninteracting Fermi gas in a three-dimensional harmonic trap [38]; (ii) inserting these quantities into the reservoir Fermi-Dirac distributions $f_{L, R}$ to compute the time derivatives of particle number and energy in each reservoir,

$$
\begin{aligned}
\frac{d N_{L, R}}{d t}= & \frac{1}{h} \int_{-\infty}^{+\infty} f_{R, L}(E) \mathcal{T}(E) d E \\
& -\frac{1}{h} \int_{-\infty}^{+\infty} f_{L, R}(E)[\mathcal{T}(E)+\mathcal{L}(E)] d E \\
\frac{d U_{L, R}}{d t}= & \frac{1}{h} \int_{-\infty}^{+\infty} E \cdot f_{R, L}(E) \mathcal{T}(E) d E \\
& -\frac{1}{h} \int_{-\infty}^{+\infty} E \cdot f_{L, R}(E)[\mathcal{T}(E)+\mathcal{L}(E)] d E
\end{aligned}
$$

and (iii) updating the particle number and energy after a numerical time step using Euler's method.

\section{EXPERIMENTAL PROCEDURE}

In this section, we present the characteristics of the experiment and of the conductance measurements. We also justify why the scattering of a photon, normally associated with a large momentum kick, can be modeled by a local particle loss without increasing the total energy of the system.

\section{A. Experimental parameters}

We prepare atoms in the geometry depicted in Fig. 1(a) of an atomic QPC consisting of two reservoirs connected by a $1 \mathrm{D}$ region where the atomic density is tunable.

We start by producing a degenerate cloud of ${ }^{6} \mathrm{Li}$ atoms in a balanced mixture of $|\downarrow\rangle$ and $|\uparrow\rangle$ (the lowest and thirdlowest hyperfine states), with a typical temperature of $T=$ $66(12) \mathrm{nK}$ and $N=1.1(1) \times 10^{5}$ atoms per spin state. Before starting the transport experiment, the magnetic field is ramped to a value of 568 and $574 \mathrm{G}$ for Fig. 3 and Figs. 4-6 where the scattering length between the two components is $a=0(7) a_{0}$ and 91(7) $a_{0}$, respectively. The atoms are thus very weakly interacting and atom-atom scattering is expected to be weak, even in the $1 \mathrm{D}$ region of the QPC.

The cloud is then shaped into two reservoirs by projecting the different optical potentials [see Fig. 1(a)]. The vertical (horizontal) confinement is provided by a beam 


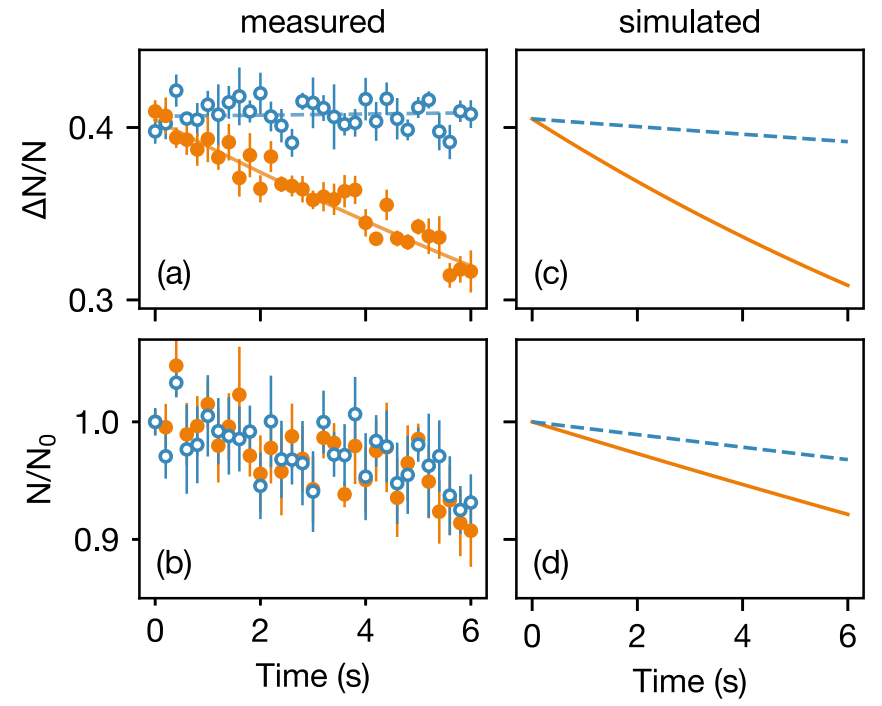

FIG. 3. Measured and simulated evolution of the relative atom number imbalance and of the atom number. (a) Experimental relative imbalance and (b) normalized atom number for spin $|\downarrow\rangle$ (orange, closed symbols) and spin $|\uparrow\rangle$ (blue, open symbols), obtained for a mean chemical potential $V_{g}+\mu_{\text {res }}=k_{B} \times 0.61(2) \mu \mathrm{K}$, with a nearresonant beam power $P_{s}=20(6) \mathrm{pW}$ corresponding to an intensity $I_{s}=0.13(4) I_{\text {sat }}$, where $I_{\text {sat }}$ is the $D_{2}$-line saturation intensity and at a scattering length $a=0$ (7) $a_{0}$. The initial atom numbers for each spin state are $N_{0, \downarrow}=117 \times 10^{3}$ and $N_{0, \uparrow}=110 \times 10^{3}$. Here and in the following, error bars correspond to the standard error of the mean of three measurements. (c) Simulation results for the relative atom number difference $\Delta N / N$ and (d) the normalized atom number with spin-dependent potential $V_{s}=k_{B} \times 0.25 \mu \mathrm{K}$, gate potential $V_{g}=k_{B} \times 0.38 \mu \mathrm{K}$, temperature $T=60 \mathrm{nK}$, and initial conditions $N_{0, \downarrow / \uparrow}=115 \times 10^{3}$ and $\Delta N_{0} / N_{0}=0.41$. Losses occur mostly for $|\downarrow\rangle$ atoms.

propagating along $x(z)$ with a waist along $y$ of $w_{z}=30.2 \mu \mathrm{m}$ $\left(w_{x}=5.9 \mu \mathrm{m}\right)$ with maximum confinement frequency $v_{z}=$ $9.03(5) \mathrm{kHz}\left[v_{x}=14.0(6) \mathrm{kHz}\right]$. The length of the QPC is mainly defined by the shortest waist of the constriction beams, namely, $w_{x}$. The mean chemical potential of the reservoirs is typically $\mu_{\mathrm{res}}=\left(\mu_{L}+\mu_{R}\right) / 2=k_{B} \times 0.23 \mu \mathrm{K}$. The density in and around the $1 \mathrm{D}$ region is tuned using an attractive gate beam of waist $w_{g}=31.8(3) \mu \mathrm{m}$ and of maximum potential $V_{g}$. This increases the local value of the chemical potential relevant for understanding transport to $V_{g}+\mu_{\text {res }}$. Inside the QPC, we add a near-resonant light beam the frequency of which can be tuned between the two resonances for states $|\uparrow\rangle$ and $|\downarrow\rangle$ of the $\mathrm{D}_{2}$ line for $\sigma^{-}$polarized light [see Figs. 2(a) and 2(b)]. This beam is shaped into a Gaussian profile with a waist of $w_{s}=2.0(1) \mu \mathrm{m}$ thanks to a digital micromirror device that allows for aberration correction and precise positioning inside the constriction [31]. Its power of $P_{s}=20(6) \mathrm{pW}$ corresponds to a peak intensity of $I_{s}=2 P_{s} / \pi w_{s}^{2}=3(1) \mathrm{W} / \mathrm{m}^{2}=$ $0.13(4) I_{\text {sat }}$. For equal and opposite detunings from the two resonances $\bar{\delta}=0$, this corresponds to a dipole potential of $V_{s}=V_{\uparrow}=-V_{\downarrow}=k_{B} \times 330(98) \mathrm{nK}$ and a photon absorption rate $\Gamma=3.1(9) \times 10^{3} \mathrm{~s}^{-1}$.

Neglecting the spatial variations of the attractive gate beam, the potential landscape $V_{\sigma}(y)$ defined in Sec. III B consists of (1) a space-dependent zero-point energy due to the

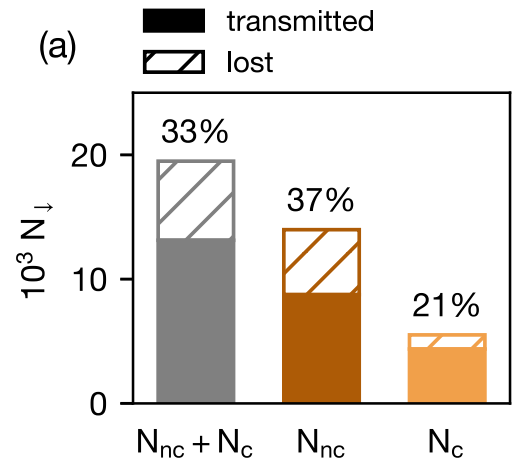

(b)

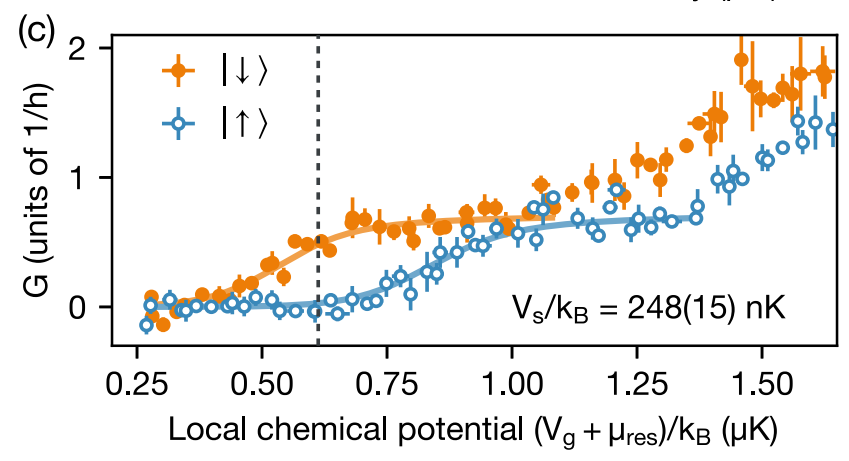

FIG. 4. Conductance plateaus are preserved at a lossy QPC. (a) Breakdown of the simulated numbers of lost and transmitted atoms as a function of their energy using the parameters of Fig. 3 integrated over a transport time of $4 \mathrm{~s}$. This highlights one reason for the robustness of transport observables. The total number of lost (transmitted) atoms (first bar of the chart) can be decomposed into two parts, shown in the filled (hatched) region: (i) those which do not contribute to transport [Eqs. (18) and (25), brown region in Fig. 1 and second bar of the chart] and (ii) those which could contribute to transport [Eqs. (17) and (24), orange region in Fig. 1 and third bar of the chart]; see text for details. The relative fraction of lost atoms in each category, indicated on top of each bar, shows that the losses are less important for the atoms participating in transport (due to their higher velocities) than for the other atoms, hence conductance is weakly affected by the losses. (b) Quasi-1D potentials along the transport direction $y$ for each spin state $V_{\sigma}(y)$. The chemical potential of Fig. 3 and panel (a) is indicated as dashed lines. (c) Conductance $G$ of each spin state at scattering length $a=91(7) a_{0}$ vs local chemical potential $V_{g}+\mu_{\text {res }}$ with a near-resonant beam intensity of $I_{s}=0.13(4) I_{\text {sat }}$. Fits by a Landauer model are shown as solid curves and indicate a spin-dependent potential of $V_{s}=k_{B} \times 0.25(2) \mu \mathrm{K}$.

$x$ and $z$ confinement

$$
V_{0}(y)=\frac{1}{2} h v_{z} e^{-y^{2} / w_{z}^{2}}+\frac{1}{2} h v_{x} e^{-y^{2} / w_{x}^{2}}
$$

where $v_{x, z}$ are the maximum confinement frequencies and $w_{x, z}$ are the waists along $y$ of the beams providing this confinement; (2) a spin-dependent potential $V_{\alpha}(y)$ defined by Eq. (5) and proportional to the near-resonant beam intensity $I(y)=I_{s} e^{-2 y^{2} / w_{s}^{2}}$,

$$
V_{\alpha}(y)=\epsilon_{\alpha} V_{s} e^{-2 y^{2} / w_{s}^{2}},
$$

with $\epsilon_{\uparrow}=+1$ and $\epsilon_{\downarrow}=-1$; and (3) an imaginary potential $i V_{\text {loss }}(y)=-i \hbar \frac{\Gamma(y)}{2}$ introduced in the effective Hamiltonian 


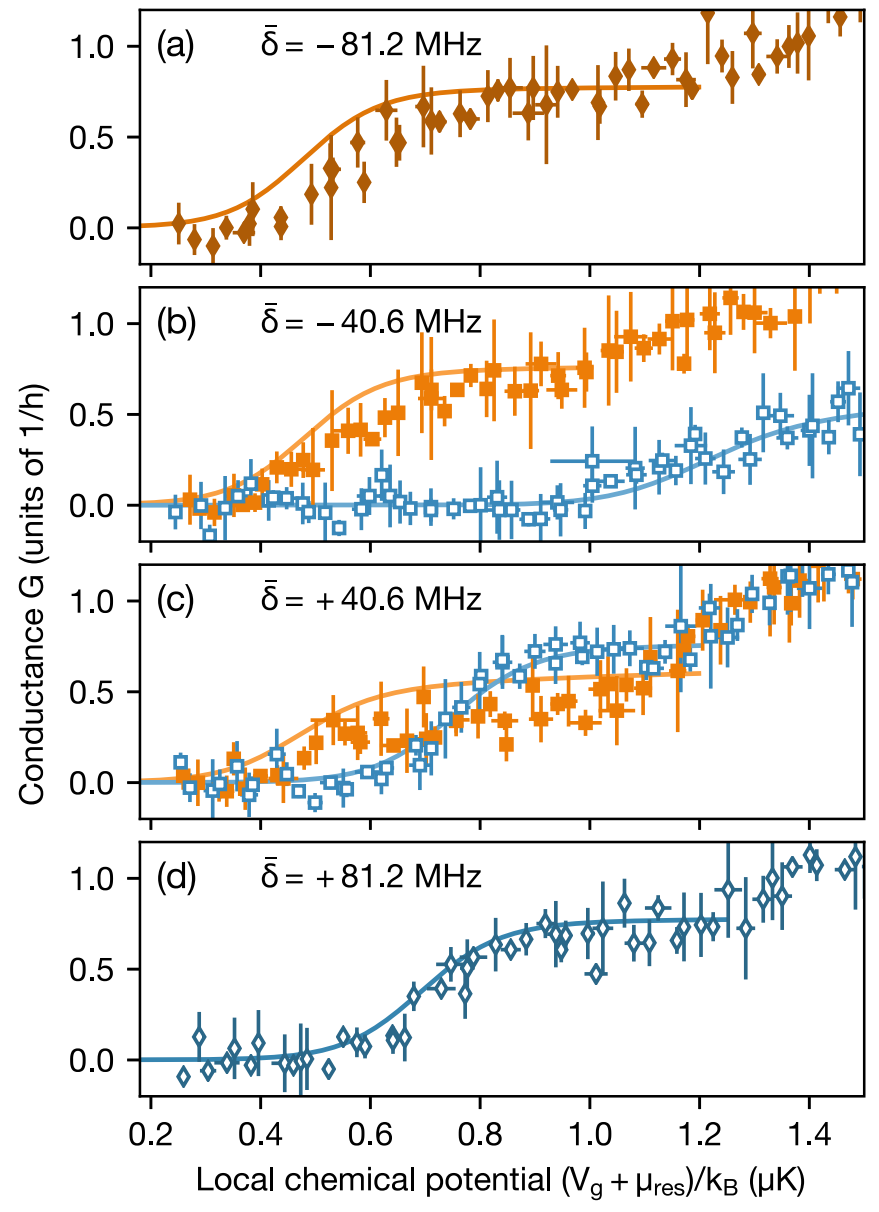

FIG. 5. Validity of the Landauer model for large dissipation. Conductance $G$ at a scattering length $a=91(7) a_{0}$ for spin $|\downarrow\rangle$ (orange, closed symbols) and spin $|\uparrow\rangle$ (blue, open symbols) with near-resonant light (a) on resonance with $|\uparrow\rangle$ at detuning $\bar{\delta}=$ $-81.3 \mathrm{MHz}$, (b) at detuning $\bar{\delta}=-40.6 \mathrm{MHz}$, (c) at detuning $\bar{\delta}=$ $40.6 \mathrm{MHz}$, and (d) on resonance with $|\downarrow\rangle$ at detuning $\bar{\delta}=81.3 \mathrm{MHz}$. Solid curves show a Landauer prediction using the fit parameters of Fig. 4(c) extended to different detunings. The different detunings at which the conductance is measured are indicated by dashed gray lines in Figs. 2(a) and 2(b).

(11), describing losses due to the scattering rate $\Gamma(y)$ defined by Eq. (6) that is proportional to $I(y)$.

At the end of an experimental cycle, the atomic density of the cloud is recorded using absorption imaging. The density profile is then fitted to the equation of state of the noninteracting Fermi gas to extract the atom number, temperature, and chemical potential of each reservoir.

\section{B. Transport measurement}

Transport experiments are performed by introducing an initial atom number between the two reservoirs and letting the system relax to equilibrium via the flow of particles of each internal state through the ballistic channel. Typically, we prepare for each spin state atom number differences of $\Delta N(0)=45(3) \times 10^{3}$. As demonstrated in previous works, in the absence of dissipation, this system is very well described using Landauer-Büttiker theory [27].
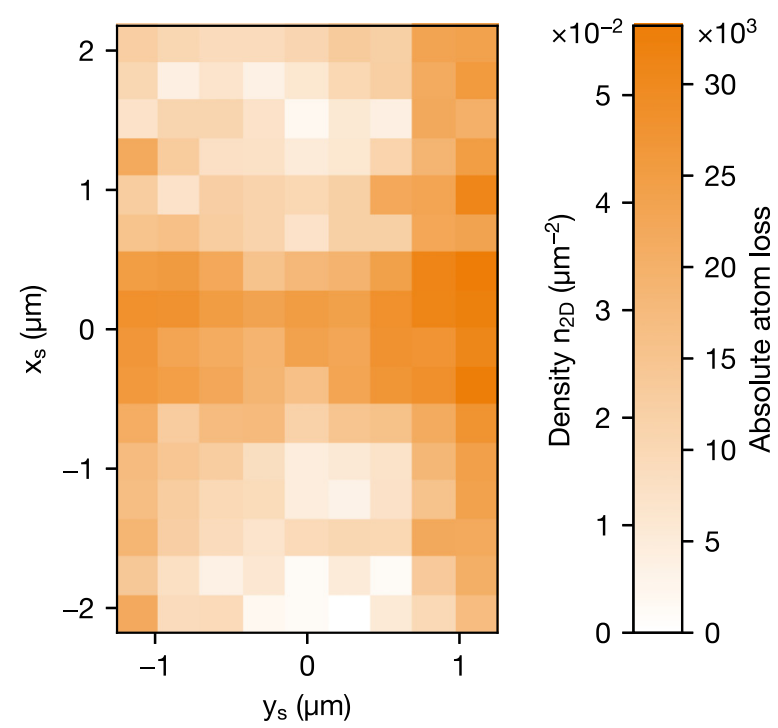

FIG. 6. Dissipative scanning gate microscopy. Integrated atom loss in state $|\downarrow\rangle$ after a time $\Delta t=4 \mathrm{~s}$ as a function of the nearresonant beam position $\left(x_{s}, y_{s}\right)$ and corresponding two-dimensional density $n_{2 \mathrm{D}}$, showing a high-resolution map of the QPC. The exact value of $n_{2 \mathrm{D}}$ should be obtained by deconvolving this map with the spatial profile of the near-resonant beam, which is beyond the scope of this paper. The near-resonant beam has a detuning of $\bar{\delta}=40 \mathrm{MHz}$, a narrower waist of $w_{s}=1.02(5) \mu \mathrm{m}$, and a power $P_{s}=4(1) \times$ $10^{1} \mathrm{pW}$, corresponding to a peak intensity of $I_{s}=24(8) \mathrm{W} / \mathrm{m}^{2}$ and therefore a large photon absorption rate $\Gamma=9(3) \times 10^{4} \mathrm{~s}^{-1}$ for state $|\downarrow\rangle$.

When the temperature difference between the reservoirs is zero, i.e., $\Delta T=0$, the particle current through the QPC is linear in the chemical potential between the reservoirs following Eq. (21). The linear approximation is valid for the weak interaction strengths considered here [39]. They also ensure that spin drag is negligible and that biases, currents, and transport coefficients can be treated independently for each spin $\sigma \in\{\uparrow, \downarrow\}$ (omitted in the rest of the subsection).

The chemical potential for each reservoir $r \in\{L, R\}$ and each spin can be furthermore expanded to first order around the atom number at $t=0, d \mu_{r}=d N_{r} / \kappa_{r}$, where $\kappa_{r}$ is the compressibility of one reservoir. Equation (21) can then be simplified to a closed first-order differential equation in the atom number difference $\Delta N$. This difference is an exponentially decreasing function of time, showing that the atomic QPC is the analog of an $R C$ circuit for neutral atoms, with a time constant

$$
\tau=G\left(\frac{1}{\kappa_{\mathrm{L}}}+\frac{1}{\kappa_{\mathrm{R}}}\right) \approx \frac{2 G}{\kappa},
$$

where $\kappa$ is the reservoir compressibility at global equilibrium for a mean atom number $\bar{N}=\left(N_{L}+N_{R}\right) / 2$ and temperature $T$ computed from the trap geometry and the noninteracting Fermi gas properties. The timescale $\tau$ is extracted from the atom number difference $\Delta N / N=\left(N_{L}-N_{R}\right) /\left(N_{L}+N_{R}\right)$ at $t=0 \mathrm{~s}$ and after a fixed transport time $t=4 \mathrm{~s}$, which in turn yields the conductance $G$.

Using thermodynamical variables such as $T, \mu$, or $\kappa$ is possible as long as the reservoirs can be described by thermal 
states, which is valid when the scattering time in the reservoirs $\tau_{s}$ is short compared to the characteristic timescale of the transport $\tau$. The scattering time can be approximated by $\tau_{s}=$ $1 / \bar{n} \sigma v_{F}$, where $\bar{n}$ is the peak density and $v_{F}$ is the Fermi velocity taken at the trap center, and $\sigma$ is the interatomic scattering cross section. For a scattering length of $a=91 a_{0}, \tau_{s}=0.08 \mathrm{~s}$ while $\tau$ is on the order of several seconds for a conductance around $1 / h$. In the absence of atom-atom scattering for $a=$ 0 (7) $a_{0}$, even though this assumption should formally break down, we expect the reservoirs to remain approximated by thermal states since atom number variations due to transport and losses are smaller than $10 \%$ per reservoir. Such an agreement between observables in thermalized and nonthermalized regimes was already noted in our previous experimental work [27], and is furthermore supported by theoretical studies on the validity of the Landauer formalism both in presence of incoherent baths or in a complete microcanonical picture [40].

\section{Loss mechanisms}

When an atom scatters a near-resonant photon, it gets a large kinetic energy corresponding to the two momentum kicks associated with the absorption and spontaneous reemission of the photon. It is therefore not lost immediately but travels through the cloud of trapped atoms and can interact with them. In this subsection, we show that an atom that underwent a scattering event can be considered as lost for moderate interaction strengths between the two component of the gas. This requires us to study (i) the single-particle dynamics after a photon scattering event as well as the subsequent scattering with (ii) atoms inside the wire and (iii) atoms in the reservoir.

Atoms that absorb a photon reemit it spontaneously at a rate $\Gamma_{0}=36.9 \mu \mathrm{s}^{-1}$ during which they move by less than $1 \mathrm{~nm}$. The imparted recoil energy $E_{R}=(h / \lambda)^{2} / 2 m=k_{B} \times$ $3.54 \mu \mathrm{K}$ is smaller than the potential barrier imposed by the beams defining the QPC, equal to $k_{B} \times 48 \mu \mathrm{K}$ in the $z$ direction and $k_{B} \times 7 \mu \mathrm{K}$ in the $x$ direction. Atoms therefore do not escape the QPC laterally and are projected to a superposition of transverse QPC modes, described by the Lamb-Dicke parameter $\eta_{z}=\sqrt{E_{R} / h \nu_{z}}=2.86(1)$ for a recoil momentum transfer along $z$ and $\eta_{x}=\sqrt{E_{R} / h v_{x}}=2.29(4)$ along $x$. They are, however, directed towards the reservoirs along the nonconfined direction $y$.

Once the atom has scattered a photon, it is projected into an excited state of the 2D harmonic oscillator the quantum number of which is $n_{x, z} \simeq \eta_{x, z}^{2}$ on average and can still scatter with another low-energy atom in the wire. With strong harmonic confinement in two directions of space, the $s$ wave scattering properties can be modified by confinement induced resonances [41]. Using [42], we can compute the transmission probability associated to the scattering event of an atom in $\left(n_{x}, n_{z}\right)$ with an atom in the ground state of the two transverse harmonic oscillator at a relative momentum of $k_{R}=2 \pi / \lambda$. The probability that the atoms are transmitted without changing their oscillator and momentum states is equal to $T=1-6.1 \times 10^{-4}$, which has to be exponentiated by the number of potential atom-atom scattering events in the channel. There is therefore less than $0.4 \%$ chance that such a collision happens with any atom in the wire, hence scattering events between the energetic particle and the atoms in the channel can be neglected.

When an atom at the recoil velocity enters the threedimensional reservoirs, the relevant quantity to consider is its mean free path $\ell=1 / \bar{n} \sigma$. For the largest interaction strength considered here, its value is $\ell=3.26(5) \mathrm{mm}$, much larger than the reservoir size of approximately $0.2 \mathrm{~mm}$. Since the recoil energy is larger than the depth $V_{\text {trap }}=k_{B} \times 0.55 \mu \mathrm{K}$ of the optical trap defining the reservoirs, scattered atoms eventually escape the system and do not contribute to a global energy increase for the weak interactions.

Therefore, for weak $s$-wave interactions, an atom which has scattered a photon can be considered as lost and the formalism of Sec. III applies.

\section{EXPERIMENTAL INVESTIGATION OF TRANSPORT WITH LOSSES}

We now compare the measurements to the models of Secs. II and III. First, we compare the experimental time evolution of the total atom number and relative imbalance for a fixed value of the detuning to the simulation. From this evolution, we can extract the conductance of the QPC and demonstrate that the expected conductance plateaus remain visible even at large local chemical potential, with a value renormalized by the loss probability. We then vary the detuning of the near-resonant tweezer to show the validity of the LandauerBüttiker model even when the tweezer is brought on resonance with one of the two spin states. Last, varying the position of the tweezer in the channel generates losses proportional to the local atomic density, allowing us to map it in and around the QPC.

\section{A. Atom number evolution in the reservoirs}

In a first measurement, we study the evolution of the atom number in each reservoir as a function of time both experimentally and numerically.

With the experimental setup and parameters described in Sec. IV A, the photon scattering rates are equal for the two internal states while the potentials are of equal and opposite magnitude, as illustrated in Figs. 2(a) and 2(b). The relative imbalance $\Delta N / N=\left(N_{L}-N_{R}\right) /\left(N_{L}+N_{R}\right)$ and the normalized atom number $N / N_{0}=\left(N_{L}+N_{R}\right) /\left[N_{L}(t=0)+N_{R}(t=\right.$ $0)]$ are recorded over $6 \mathrm{~s}$ at a magnetic field of $568 \mathrm{G}$ where the $s$-wave scattering length is $a=0(7) a_{0}$. The results, presented in Figs. 3(a) and 3(b), illustrate that the spin-dependent potential acts as a repulsive barrier for the $|\uparrow\rangle$ state: the fitted current $I_{\uparrow}=-19 \pm 85 \mathrm{~s}^{-1}$ vanishes while the one for the $|\downarrow\rangle$ state has a finite value of $I_{\downarrow}=833 \pm 98 \mathrm{~s}^{-1}$ corresponding to a conductance of $G_{\downarrow}=0.45(4) / h$. In spite of a maximal photon scattering rate $\Gamma_{s}=2.3(8) \times 10^{3} \mathrm{~s}^{-1}$ at the center of the tweezer, the atom losses are moderate since they represent less than $10 \%$ of the total atom number.

This experiment is reproduced by a numerical simulation following Sec. IIID using the parameters obtained experimentally (near-resonant beam intensity, initial atom number, initial imbalance, and temperature). The numerical results shown in Figs. 3(c) and 3(d) are largely consistent with the experimental results, indicating that for moderate values of the 
conductance, neglecting fluctuations, extending the LandauerBüttiker model and integrating the time evolution of the cloud properties as in Sec. III constitute a valid approach. The simulation also indicates that losses should be smaller for $|\uparrow\rangle$ atoms which are blocked by the spin-dependent potential and therefore have lower densities in the dissipative region, with a $5 \%$ difference in atom number for both spin states. This effect nevertheless remains elusive in the experimental data because of the uncertainty in the measured atom numbers since this difference is on the order of our preparation noise in terms of atom numbers.

\section{B. Preserving the conductance plateaus at a lossy QPC}

In Sec. III, we have showed that transport observables are sensitive only to scattering at energies close to the Fermi level which concerns a small fraction of all atoms subject to nearresonant light. Conductance is therefore expected to be robust against losses. As illustrated in Fig. 1(b) (brown areas), most of the losses actually concern atoms that are below the Fermi surface (25) and therefore do not affect conductance.

This is verified by integrating the simulation results of Fig. 3 over $t=4 \mathrm{~s}$ of transport time. We thus obtain the number of atoms transmitted or lost and participating or not in transport (subscripts "c" and "nc"),

$$
N_{\mathrm{c} / \mathrm{nc}}^{\text {trans } / \text { lost }}=\int_{0}^{t} \dot{N}_{\mathrm{c} / \mathrm{nc}}^{\text {trans } / \text { lost }} d t^{\prime},
$$

using the quantities defined in Secs. III C and III D. We then extract the total number of particle participating in transport $N_{\mathrm{c}}=N_{\mathrm{c}}^{\text {lost }}+N_{\mathrm{c}}^{\text {trans }}$ and nonparticipating in transport $N_{\mathrm{nc}}=$ $N_{\mathrm{nc}}^{\text {lost }}+N_{\mathrm{nc}}^{\text {trans }}$. The value of these quantities and their sum is represented in Fig. 4(b) for the $|\downarrow\rangle$ state: while $33 \%$ of the particles flowing through the dissipative region are lost, only $21 \%$ of the particles contributing to net transport are dissipated due to their larger velocities compared with the noncontributing particles.

Recording conductance as a function of local chemical potential demonstrates that plateaus are still visible at the lossy QPC, as shown in Fig. 4(c). We fit the conductances of both states with the Landauer model [solid curves in Fig. 4(c)] described in Sec. III. The relevant model parameters are summarized by the following function:

$$
G_{\sigma}(x)=A G_{\sigma}^{\mathrm{th}}\left(x-\mu_{0}, T, \bar{\delta}, V_{s}\right),
$$

where $G_{\sigma}^{\text {th }}$ is obtained using the Landauer-Büttiker formula for the $|\downarrow\rangle$ spin. The fixed parameters are the temperature $T$ fixed to its measured value and the detuning relative to the mean resonance frequency $\bar{\delta}=0$. The fitted parameters are a small chemical potential offset $\mu_{0}=k_{B} \times 0.06(2) \mu \mathrm{K}$ and a scaling factor capturing the decrease of the extracted conductance below $1 / h$ due to remaining weak thermoelectric effects $A=0.78(3)$. This yields an experimental value of the spin-dependent potential of $V_{s}=k_{B} \times 0.25(2) \mu \mathrm{K}$, compatible with the theoretical value of $k_{B} \times 0.29(11) \mu \mathrm{K}$ for an intensity of $3(1) \mathrm{W} / \mathrm{m}^{2}$

In a Landauer picture valid for weak interactions, these losses contribute to decreasing the conductance by the scattering probability. This probability is computed to be $21 \%$ in
Fig. 4(a) and is consistent with the decrease of the conductance plateau from $G=0.84(1) / h$ to $0.72(3) / h$.

\section{Varying the loss rates}

To explore the validity range of this Landauer model with losses presented in Sec. III, we extend our conductance measurements to different tweezer detunings $\bar{\delta}$ relative to the mean resonance frequency $\left(v_{\uparrow}+v_{\downarrow}\right) / 2$ at fixed intensity $I_{s}=0.13(4) I_{\text {sat }}$ and at an interaction strength of 91(7) $a_{0}$.

As shown in Figs. 2(a) and 2(b), this affects both spindependent dipole potential $V_{\sigma}$ and photon scattering rate $\Gamma_{\sigma}$, and allows us to change the latter by more than three orders of magnitude. We explore detunings ranging from the tweezer being resonant with $|\uparrow\rangle$ at $\bar{\delta}=-81.2 \mathrm{MHz}$ in Fig. 5(a) to the $|\downarrow\rangle$ resonance at $\bar{\delta}=81.2 \mathrm{MHz}$ in Fig. 5(d). Bringing the tweezer on resonance with one of the two states leads to its entire loss after $4 \mathrm{~s}$, while the other nonresonant state still displays quantized conductance [Figs. 5(a) and 5(d)]. The applicability of the Landauer theory highlights that the coupling between spins is negligible at this scattering length.

In addition, tuning the frequency by $\bar{\delta}=-40.6 \mathrm{MHz}$ towards the resonance of $|\uparrow\rangle$ leads to a shift of the conductance curve towards higher chemical potentials [Fig. 5(b), blue], since the repulsive potential barrier and scattering rate for that state are increased. Meanwhile, the conductance of $|\downarrow\rangle$ [Fig. 5(b), orange] approaches the one measured in the absence of near-resonant light.

The reverse trend is observed with a detuning $\bar{\delta}=$ $+40.6 \mathrm{MHz}$, where the conductance of $|\downarrow\rangle$ is clearly reduced due to increased losses [Fig. 5(c)]. These measurements show good agreement with the previous Landauer model without having to add any fit parameters.

The good agreement demonstrates the applicability of the Landauer-Büttiker formula over a wide range of dissipation strengths.

\section{Scanning gate microscopy with losses}

We have so far studied the effect of dissipation in the transport structure, developing an extension to the Landauer model; now we can further use the dissipative beam as a density probe. The near-resonant tweezer is corrected for aberrations using a digital micromirror device in Fourier configuration. A discretized grating therefore controls the phase front of the beam which determines its position inside the QPC at the submicron level. We take advantage of this precise positioning by recording the total atom loss as a function of the tweezer position to infer the local atomic density. We perform this experiment with a vanishing atom imbalance between the reservoirs.

To model the situation, we assume that the atomic density is two dimensional and time independent. These assumptions hold provided that the density variations along the $z$ direction are small compared to the Rayleigh length of the nearresonant beam $z_{R}=\pi w_{s}^{2} / \lambda=4.9(3) \mu \mathrm{m}$, and that the atom losses remain small relative to the total atom number. 
The atom losses integrated over the time interval $\Delta t$ during which photon scattering occurs can be written as

$$
\begin{gathered}
N(0)-N(t)=\Delta t \int d x d y \Gamma(x, y) n_{2 \mathrm{D}}(x, y) d x d y \\
\text { with } \Gamma(x, y)=\Gamma_{s} e^{-2\left[\left(x-x_{s}\right)^{2}+\left(y-y_{s}\right)^{2}\right] / w_{s}^{2}} .
\end{gathered}
$$

Losses are proportional to the atomic density convolved with the Gaussian intensity profile of the near-resonant beam centered at position $\left(x_{s}, y_{s}\right)$. In the limit where its Gaussian waist $w_{s}$ is small with respect to the variations of the atomic density, it can be approximated by a Dirac function and the local density is given by

$$
n_{2 \mathrm{D}}\left(x_{s}, y_{s}\right)=\frac{2}{\pi w_{s}^{2}} \frac{N(0)-N(t)}{\Gamma_{s} \Delta t} .
$$

The near-resonant beam also acts as an attractive beam, slightly shifting the local chemical potential.

As long as the chemical potential shift is small compared to the mean value, and as the Gaussian waist is small, repeating measurements of atom losses for different positions of the near-resonant beam allows us to retrieve a map of the twodimensional density, as shown in Fig. 6. This measurement is conceptually similar to scanning probe microscopy in solidstate physics, and complements previous imaging techniques for quantum gases using absorption imaging, a focused electron beam [18], or a conservative optical potential in a transport geometry [43]. The dissipative scanning gate technique is favorable over standard absorption imaging because the resolution is limited by the minimal displacement possible with the near-resonant beam (below $0.3 \mu \mathrm{m}$ ), which is smaller than the resolution of the optical system used to project the potential (of $0.7 \mu \mathrm{m}$ ).

\section{CONCLUSION}

Adding a near-resonant beam at an atomic QPC leads to different potentials and scattering rates for the different spin states. These values can be computed from the atomic polarizability and used to express the transmission and loss probabilities of each particle traveling through the point contact. This required extending the Landauer-Büttiker formalism to a situation with losses which reproduces the measurement of the conductance, where a plateau remains visible although its value is decreased with respect to the quantum of conductance $1 / h$. It is also possible to integrate the results of this model to express the time evolution of the atom number and relative imbalance between the reservoirs of the two-terminal geometry. Finally, the atom losses can be related to the atomic density at the position of the near-resonant tweezer, which can therefore act as a "dissipative scanning gate microscope" for ultracold gases.

The ability to engineer dissipation in a transport experiment opens the possibility to study the competition between losses and coherent transport by investigating the continuous Zeno effect [44] or the modification of transport through a mesoscopic, dissipative lattice. Ultracold atoms also allow us to vary the $s$-wave interaction strength up to the point where a paired superfluid is formed. There, characteristic signatures of transport through a tunnel barrier can also be strongly influenced by the presence of dissipation [45].

\section{ACKNOWLEDGMENTS}

We thank T. Giamarchi, L. Glazman, H. Moritz, H. Ott, and A.-M. Visuri for helpful discussions, and J.-P. Brantut, R. Citro, M. Landini, and K. Viebahn for their critical reading of the manuscript. We acknowledge the Swiss National Science Foundation (Grants No. 182650 and No. NCCR-QSIT) and European Research Council advanced grant TransQ (Grant No. 742579) for funding. L.C. is supported by an ETH Zurich Postdoctoral Fellowship, the Marie Curie Actions for People COFUND program, and the European Union Horizon 2020 Marie Curie TopSpiD program (Grant No. 746150).
[1] R. Balian, From Microphysics to Macrophysics: Methods and Applications of Statistical Physics (Springer, New York, 2007).

[2] E. Schrödinger, Die Naturwissenschaften 23, 1 (1935).

[3] J.-M. Raimond and S. Haroche, Exploring the Quantum (Oxford University, New York, 2006).

[4] M. Müller, S. Diehl, G. Pupillo, and P. Zoller, Advances in Atomic, Molecular, and Optical Physics, Vol. 61 (Elsevier, New York, 2012), pp. 1-80.

[5] A. J. Daley, Adv. Phys. 63, 77 (2014).

[6] H.-P. Breuer and F. Petruccione, The Theory of Open Quantum Systems (Oxford University, New York, 2002).

[7] A. Caldeira and A. J. Leggett, Ann. Phys. 149, 374 (1983).

[8] A. J. Leggett, S. Chakravarty, A. T. Dorsey, M. P. Fisher, A. Garg, and W. Zwerger, Rev. Mod. Phys. 59, 1 (1987).

[9] N. Moiseyev, Non-Hermitian Quantum Mechanics (Cambridge University, Cambridge, England, 2011).

[10] M.-A. Miri and A. Alù, Science 363, eaar7709 (2019).

[11] M. Büttiker, Phys. Rev. B 33, 3020 (1986).

[12] S. Datta, Phys. Rev. B 40, 5830 (1989).
[13] F. Sols, Ann. Phys. (NY) 214, 386 (1992).

[14] T. Tomita, S. Nakajima, I. Danshita, Y. Takasu, and Y. Takahashi, Sci. Adv. 3, e1701513 (2017).

[15] N. Syassen, D. M. Bauer, M. Lettner, T. Volz, D. Dietze, J. J. Garcia-Ripoll, J. I. Cirac, G. Rempe, and S. Dürr, Science 320, 1329 (2008).

[16] A. Amico, F. Scazza, G. Valtolina, P. E. S. Tavares, W. Ketterle, M. Inguscio, G. Roati, and M. Zaccanti, Phys. Rev. Lett. 121, 253602 (2018).

[17] M. Schemmer and I. Bouchoule, Phys. Rev. Lett. 121, 200401 (2018).

[18] T. Gericke, P. Wurtz, D. Reitz, T. Langen, and H. Ott, Nat. Phys. 4, 949 (2008).

[19] G. Barontini, R. Labouvie, F. Stubenrauch, A. Vogler, V. Guarrera, and H. Ott, Phys. Rev. Lett. 110, 035302 (2013).

[20] P. Wessels, B. Ruff, T. Kroker, A. K. Kazansky, N. M. Kabachnik, K. Sengstock, M. Drescher, and J. Simonet, Commun. Phys. 1, 32 (2018). 
[21] T. Pfau, S. Spälter, C. Kurtsiefer, C. R. Ekstrom, and J. Mlynek, Phys. Rev. Lett. 73, 1223 (1994).

[22] Y. S. Patil, S. Chakram, and M. Vengalattore, Phys. Rev. Lett. 115, 140402 (2015).

[23] R. Bouganne, M. B. Aguilera, A. Ghermaoui, J. Beugnon, and F. Gerbier, arXiv:1905.04808 (2019).

[24] R. Labouvie, B. Santra, S. Heun, and H. Ott, Phys. Rev. Lett. 116, 235302 (2016).

[25] A. Müllers, B. Santra, C. Baals, J. Jiang, J. Benary, R. Labouvie, D. A. Zezyulin, V. V. Konotop, and H. Ott, Sci. Adv. 4, eaat6539 (2018).

[26] S. Krinner, T. Esslinger, and J.-P. Brantut, J. Phys.: Condens. Matter 29, 343003 (2017).

[27] S. Krinner, D. Stadler, D. Husmann, J.-P. Brantut, and T. Esslinger, Nature (London) 517, 64 (2015).

[28] R. Landauer, IBM J. Res. Dev. 1, 223 (1957).

[29] M. Büttiker, Phys. Rev. Lett. 57, 1761 (1986).

[30] Y. Imry, Introduction to Mesoscopic Physics (Oxford University, New York, 2002), Vol. 2.

[31] P. Zupancic, P. M. Preiss, R. Ma, A. Lukin, M. E. Tai, M. Rispoli, R. Islam, and M. Greiner, Opt. Express 24, 13881 (2016).

[32] M. Lebrat, S. Häusler, P. Fabritius, D. Husmann, L. Corman, and T. Esslinger, Phys. Rev. Lett. 123, 193605 (2019).
[33] M. E. Gehm, Ph.D. thesis, Duke University, 2003.

[34] I. Carusotto and C. Ciuti, Rev. Mod. Phys. 85, 299 (2013).

[35] E. Hairer, S. P. Noersett, and G. Wanner, Solving Ordinary Differential Equations I: Nonstiff Problems: With 105 Figures (Springer-Verlag, Berlin, 1987).

[36] S. Datta, Electronic Transport in Mesoscopic Systems (Cambridge University, Cambridge, England, 1997).

[37] C. Grenier, C. Kollath, and A. Georges, C. R. Phys. 17, 1161 (2016).

[38] G. Su, J. Chen, and L. Chen, Phys. Lett. A 315, 109 (2003).

[39] S. Krinner, M. Lebrat, D. Husmann, C. Grenier, J.-P. Brantut, and T. Esslinger, Proc. Natl. Acad. Sci. USA 113, 8144 (2016).

[40] C.-C. Chien, M. Di Ventra, and M. Zwolak, Phys. Rev. A 90, 023624 (2014).

[41] M. Olshanii, Phys. Rev. Lett. 81, 938 (1998).

[42] M. G. Moore, T. Bergeman, and M. Olshanii, J. Phys. IV France 116, 69 (2004).

[43] S. Häusler, S. Nakajima, M. Lebrat, D. Husmann, S. Krinner, T. Esslinger, and J.-P. Brantut, Phys. Rev. Lett. 119, 030403 (2017).

[44] H. Fröml, A. Chiocchetta, C. Kollath, and S. Diehl, Phys. Rev. Lett. 122, 040402 (2019).

[45] F. Damanet, E. Mascarenhas, D. Pekker, and A. J. Daley, Phys. Rev. Lett. 123, 180402 (2019). 